\title{
Tunneling in quantum cosmology and holographic SYM theory
}

\author{
Kazuo Ghoroku, ${ }^{1, *}$ Yoshimasa Nakano, ${ }^{\dagger}$ Motoi Tachibana, ${ }^{2, \hbar}$ and Fumihiko Toyoda ${ }^{3, \S}$ \\ ${ }^{1}$ Fukuoka Institute of Technology, Wajiro, Fukuoka 811-0295, Japan \\ ${ }^{2}$ Department of Physics, Saga University, Saga 840-8502, Japan \\ ${ }^{3}$ Faculty of Humanity-Oriented Science and Engineering, Kinki University, Iizuka 820-8555, Japan
}

(Received 11 December 2017; published 29 March 2018)

\begin{abstract}
We study the time evolution of the early Universe, which is developed by a cosmological constant $\Lambda_{4}$ and supersymmetric Yang-Mills (SYM) fields in the Friedmann-Robertson-Walker space-time. The renormalized vacuum expectation value of the energy-momentum tensor of the SYM theory is obtained in a holographic way. It includes a radiation of the SYM field, parametrized as $C$. The evolution is controlled by this radiation $C$ and the cosmological constant $\Lambda_{4}$. For positive $\Lambda_{4}$, an inflationary solution is obtained at late time. When $C$ is added, the quantum mechanical situation at early time is fairly changed. Here we perform the early time analysis in terms of two different approaches, (i) the Wheeler-DeWitt equation and (ii) Lorentzian path integral with the Picard-Lefschetz method by introducing an effective action. The results of two methods are compared.
\end{abstract}

DOI: $10.1103 /$ PhysRevD.97.066027

\section{INTRODUCTION}

The holographic approach has been extended to supersymmetric Yang-Mills (SYM) theory in FriedmannRobertson-Walker (FRW) space-time in Refs. [1-3]. There, the vacuum expectation value of the energy-momentum tenser of the SYM fields, $\left\langle T_{\mu \nu}^{\mathrm{SYM}}\right\rangle$, has been obtained, and it has been used to study the dynamical properties of the SYM theory in FRW space-time. On the other hand, quantum and classical cosmology with a CFT has been studied in terms of this $\left\langle T_{\mu \nu}^{\mathrm{SYM}}\right\rangle$ [4-9].

Here, we develop the quantum cosmology in the system with $\left\langle T_{\mu \nu}^{\mathrm{SYM}}\right\rangle$. In both quantum approaches via the path integration and via the Wheeler-DeWitt (WDW) equation, we start from an action of the theory. In the FRW spacetime, holographically obtained $\left\langle T_{\mu \nu}^{\mathrm{SYM}}\right\rangle$ is composed of the loop corrections of the SYM theory and the so-called dark radiation ${ }^{1}$ parametrized by $C$, which is interpreted as the radiation of SYM fields [5]. This stress tensor cannot be derived from a general coordinate transformation invariant action.

\footnotetext{
*gouroku@dontaku.fit.ac.jp

ynakano@kyudai.jp

motoi@cc.saga-u.ac.jp

§toyoda@fuk.kindai.ac.jp

${ }^{1}$ This term has been originally introduced in $[10,11]$ and studied in $[12,13]$ in the context of the brane universe.
}

Published by the American Physical Society under the terms of the Creative Commons Attribution 4.0 International license. Further distribution of this work must maintain attribution to the author(s) and the published article's title, journal citation, and DOI. Funded by SCOAP ${ }^{3}$.
Therefore, in performing quantum cosmology with a CFT, we need an effective action that can lead to the Einstein equation including the $\left\langle T_{\mu \nu}^{\mathrm{SYM}}\right\rangle$. Up to now, an example of such effective action has been given for the minisuperspace of FRW space-time in Refs. $[4,7,8]$. In these, however, it is difficult to find the WDW equation or to perform the path integral due to the high nonlinearity. Here we propose a new and simple effective action, which reserves the essential property of the original theory. Then, it becomes possible to construct the WDW equation and also to perform a Lorentzian path integral to study the propagation of the Universe [14-16].

We consider here the Einstein gravity with a cosmological constant $\Lambda_{4}$ and SYM theory (or CFT). The effective action given here is written in the same form of the starting action without the SYM theory. Namely, after integrating out SYM fields, its effect is reduced to the modification of $\Lambda_{4}$ to $\Lambda_{4}^{\text {eff }}$, which depends on the scale factor $a_{0}$ and $C$. The dark radiation $C$ coming from SYM plays an important role in this effective action at small $a_{0}$ of the FRW metric.

The validity of the Lorentzian path integral has been shown in [14] for the gravity with $\Lambda_{4}$. A relevant path in the complex plane of the lapse field could provide a correct propagator of the Universe. This propagator implies an appropriate boundary condition in solving the tunneling amplitude via the WDW equation. When the dark radiation is absent, $\Lambda_{4}^{\text {eff }}$ is a constant although it is smaller than the original $\Lambda_{4}$. So there is no qualitative change in this case even if we consider CFT. As a result, it is also possible to estimate the wave function of the Universe, which is created from nothing with no boundary condition, by the 
Lorentzian path integral via a different path as shown in [17].

On the other hand, when the dark radiation exists, $\Lambda_{4}^{\text {eff }}$ is written as a function of $a_{0}$. Then, the dynamical situation at early time is drastically changed. In this case, the scenario is changed as follows: First, the Universe is generated as a small sized sphere of the radiation of SYM fields, and secondly it reappears as an inflationary universe after the tunneling process.

Our purpose is to investigate this tunneling behavior by the two methods. At first, we study by using the WDW equation, which is derived from our effective action, by imposing an appropriate boundary condition by hand. In the second, we execute the Lorentzian path-integral based on the Picard-Lefschetz theory to obtain the propagator, as given in [14].

As shown in [14] for the case of the model without SYM theory, namely, for $C=0$, we find that this method provides the semiclassical tunneling factor that is equivalent with the one obtained by the WDW equation method. In the case of $C>0$, since $\Lambda_{4}^{\text {eff }}$ becomes complicated, we consider a simplified model to assure this point. Then we find the validity of the Lorentzian path-integral method. It could give the tunneling amplitude, which is also obtained by solving the WDW equation with an appropriate boundary condition. Other interesting points found in this path-integral method are discussed and some speculations are given.

The outline of this paper is as follows. In the next section, a gravitational model with SYM theory is given and the Einstein equations in the FRW space-time are given. They are solved at large $a_{0}(t)$, and why quantum cosmology is necessary at small $a_{0}(t)$ is explained for small $C$. In Sec. III, a tractable effective action corrected by SYM theory is proposed. By using this action, quantum cosmological solutions at small $a_{0}(t)$ are shown through the WDW equation in Sec. IV and through the Lorentzian pathintegral method in Sec. V. Summary and discussions are given in the final section.

\section{COSMOLOGY DRIVEN BY CFT}

Here we consider a model where the matter part is dominated by the $\mathcal{N}=4 \mathrm{SYM}$ field with the gauge group $S U(N)$. The four-dimensional action is given as

$$
S=\int d^{4} x \sqrt{-g}\left\{\frac{1}{2 \kappa_{4}^{2}}\left(R_{4}-2 \Lambda_{4}\right)\right\}+S_{\mathrm{SYM}},
$$

where $\kappa_{4}^{2} \equiv 8 \pi G_{4}$ and $\Lambda_{4}$ denote the four-dimensional gravitational constant and cosmological constant, respectively. $S_{\text {SYM }}$ is the action for the $\mathcal{N}=4$ SYM theory. After integrating out all SYM fields under the FRW metric [with a scale parameter $a_{0}(t)$ ], the equation of motion for $a_{0}(t)$ is obtained by the Einstein equation

$$
R_{\mu \nu}-\frac{1}{2} R g_{\mu \nu}+\Lambda_{4} g_{\mu \nu}=\kappa_{4}^{2}\left\langle T_{\mu \nu}^{\mathrm{SYM}}\right\rangle,
$$

where $\left\langle T_{\mu \nu}^{\mathrm{SYM}}\right\rangle$ represents the vacuum expectation value of the energy momentum tensor for the SYM field under a given background metric.

Here, Eq. (2.2) is solved with respect to $a_{0}(t)$ under the FRW background

$$
d s_{(4)}^{2}=-d t^{2}+a_{0}(t)^{2} \gamma_{i j} d x^{i} d x^{j},
$$

where the three-dimensional metric $\gamma_{i j}$ is defined as follows:

$\gamma_{i j}(x)=\delta_{i j} \gamma^{2}(x), \quad \gamma(x)=\frac{1}{1+k \frac{\bar{r}^{2}}{4 \bar{r}_{0}^{2}}}, \quad \bar{r}^{2}=\sum_{i=1}^{3}\left(x^{i}\right)^{2}$

Then two independent equations are obtained such that

$$
\begin{gathered}
\lambda \equiv\left(\frac{\dot{a}_{0}}{a_{0}}\right)^{2}+\frac{k}{a_{0}^{2}}=\frac{\Lambda_{4}}{3}+\frac{\kappa_{4}^{2}}{3}\left\langle T_{00}^{\mathrm{SYM}}\right\rangle, \\
2 \frac{\ddot{a}_{0}}{a_{0}}+\left(\frac{\dot{a}_{0}}{a_{0}}\right)^{2}+\frac{k}{a_{0}^{2}}=\Lambda_{4}-\kappa_{4}^{2}\left\langle T_{i i}^{\mathrm{SYM}}\right\rangle .
\end{gathered}
$$

Equation (2.5) is nothing but the $t t$ component of the Einstein equation, i.e., the Friedmann equation.

From (2.5) and (2.6), we obtain the following continuity equation for density $\rho$ and pressure $p$ of the SYM fields [18-21]:

$$
\dot{\rho}+3 H(\rho+p)=0, \quad H \equiv \frac{\dot{a}_{0}}{a_{0}},
$$

where the averaged energy-momentum tensor is written in terms of $\rho$ and $p$ as

$$
\left\langle T_{\mu \nu}^{\mathrm{SYM}}\right\rangle=\operatorname{diag}\left(\rho, p g_{i j}^{0}\right),
$$

with $g_{i j}^{0}=a_{0}(t)^{2} \gamma_{i j}[18]$, and

$$
\rho=3 \alpha\left(\frac{C}{4 a_{0}^{4}}+\frac{\lambda^{2}}{16}\right), \quad p=\alpha\left\{\frac{C}{4 a_{0}^{4}}-3 \frac{\lambda^{2}}{16}\left(1+\frac{2 \dot{\lambda}}{3 \lambda} a_{0}\right)\right\},
$$

where $C$ denotes the dark radiation density and $\alpha$ is a certain coupling parameter related to the CFT.

Note here that solving (2.5) and (2.6) is equivalent to solving (2.5) and (2.7) since (2.7) is derived from (2.5) and (2.6). On the other hand, (2.7) is satisfied for $\left\langle T_{\mu \nu}^{\mathrm{SYM}}\right\rangle$. Therefore, it is enough to solve Eq. (2.5) to obtain $a_{0}(t)$. 
Here, Eq. (2.5) is written by using $\lambda$ as

$$
\lambda=\frac{\Lambda_{4}}{3}+\tilde{\alpha}^{2}\left(\frac{4 C}{a_{0}^{4}}+\lambda^{2}\right)
$$

where

$$
\tilde{\alpha}^{2}=\frac{\kappa_{4}^{2}}{16} \alpha
$$

Then (2.10) is solved with respect to $\lambda$ as

$$
\lambda=\lambda_{ \pm}
$$

where

$$
\lambda_{ \pm} \equiv \frac{1 \pm \sqrt{1-4 \tilde{\alpha}^{2}\left(\frac{\Lambda_{4}}{3}+\frac{4 \tilde{\alpha}^{2} C}{a_{0}^{4}}\right)}}{2 \tilde{\alpha}^{2}} .
$$

The explicit form of (2.12) is given as ${ }^{2}$

$$
\left(\frac{\dot{a}_{0}}{a_{0}}\right)^{2}+\frac{k}{a_{0}^{2}}=\frac{1 \pm \sqrt{1-4 \tilde{\alpha}^{2}\left(\frac{\Lambda_{4}}{3}+\frac{4 \tilde{\alpha}^{2} C}{a_{0}^{4}}\right)}}{2 \tilde{\alpha}^{2}} .
$$

When we solve (2.14), we must notice the following points. At first for finite $C$, from the reality of this equation, we find that there is a minimum value of $a_{0}$ such as

$a_{0} \geq a_{0}^{\min }=\tilde{\alpha}\left(\frac{16 C}{\tilde{\Lambda}_{4}}\right)^{1 / 4}, \quad \tilde{\Lambda}_{4}=1-4 \tilde{\alpha}^{2} \frac{\Lambda_{4}}{3}$.

For the case with $a_{0}<a_{0}^{\min }$, we need some improvement of the gravitational theory. This point remains as an open problem here. While the solutions $a_{0}(t)$ for $\lambda_{+}$and $\lambda_{-}$can be connected at $a_{0}=a_{0}^{\min }$ [6], we consider here only the case with $\lambda_{-}$. The reason for this choice is as follows: In the limit $\tilde{\alpha} \rightarrow 0$, it would be natural that $\lambda \rightarrow \Lambda_{4} / 3$. While $\lambda_{+}$ diverges, $\lambda_{-}$approaches to $\Lambda_{4} / 3$ in this limit.

It is easy to find a classical solution corresponding to $\lambda_{-}$. From (2.14), the equation to be solved is given by

$\dot{a}_{0}^{2}=-k+a_{0}^{2} \frac{1-\sqrt{1-4 \tilde{\alpha}^{2}\left(\frac{\Lambda_{4}}{3}+\frac{4 \tilde{\alpha}^{2} C}{a_{0}^{4}}\right)}}{2 \tilde{\alpha}^{2}} \quad\left(\equiv-2 V\left(a_{0}\right)\right)$.

Here we consider the case of $\Lambda_{4}>0$ and $k=1$ (closed universe). At large $a_{0}$, we always find the inflationary solution, i.e.,

\footnotetext{
${ }^{2}$ We remember that $\left(\frac{\dot{a}_{0}}{a_{0}}\right)^{2}+\frac{k}{a_{0}^{2}}=\lambda$.
}

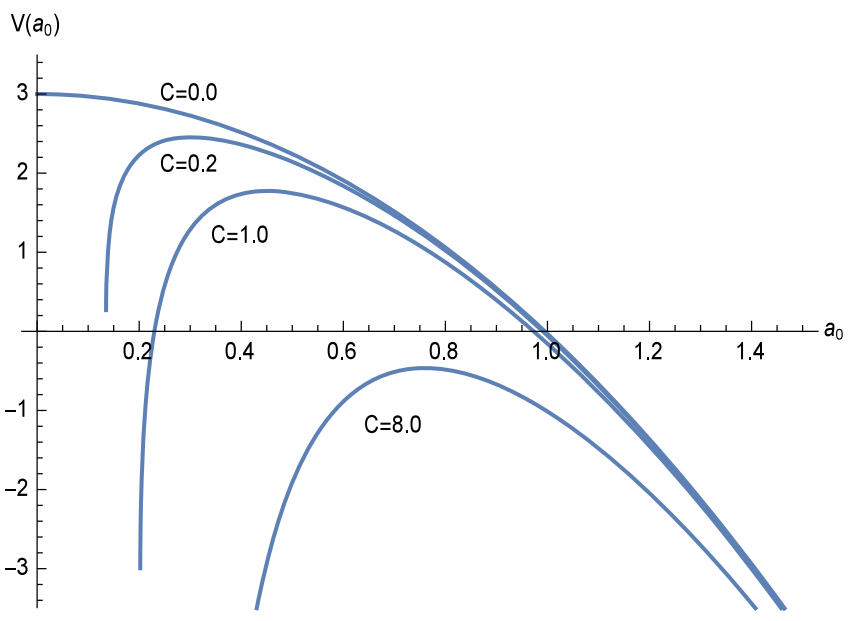

FIG. 1. Effective potential for $\Lambda_{4} / 3=1.0, k=1, \tilde{\alpha}^{2}=0.01$.

$$
a_{0}(t) \sim \exp (\gamma t), \quad \gamma=\sqrt{\frac{1-\sqrt{1-4 \tilde{\alpha}^{2} \frac{\Lambda_{4}}{3}}}{2 \tilde{\alpha}^{2}}} .
$$

Note that the expansion rate or the effective cosmological constant is suppressed by the quantum effect of the SYM theory. For small $\tilde{\alpha}$ or for small number of SYM fields, the factor $\gamma$ approaches $\sqrt{\Lambda_{4} / 3}$ as expected.

On the other hand, in the small $a_{0}$ region, we should be careful of the potential $V\left(a_{0}\right)$ defined in (2.16). Let us classify this situation into the following three types.

(a) For $0 \leq C \leq \frac{1}{4} k^{2}\left(1-4 \tilde{\alpha}^{2} \frac{\Lambda_{4}}{3}\right)$, the classical solution is restricted to the region $a_{0}^{+}<a_{0}$, where $V\left(a_{0}^{+}\right)=0$. Such a behavior is seen for the parameter region $0 \leq$ $C \leq 0.24$ in Fig. 1 .

(b) For $\frac{1}{4} k^{2}\left(1-4 \tilde{\alpha}^{2} \frac{\Lambda_{4}}{3}\right) \leq C \leq \frac{16 \tilde{\alpha}^{2} \Lambda_{4}}{3} k^{2}\left(1-4 \tilde{\alpha}^{2} \frac{\Lambda_{4}}{3}\right)$, there appears the potential barrier in the region $a_{0-} \leq a_{0} \leq$ $a_{0+}$ (see the left panel of Fig. 2). In this region, no classical solutions are allowed.

In the region $a_{0}^{\min }<a_{0}<a_{0-}$ (see the left panel of Fig. 2 again), there is a classical solution, but the solution does not provide the inflationary one. On the other hand, in the region $a_{0}>a_{0+}$, we obtain the inflationary solution. These two solutions are not connected to each other at the classical level. But they can be connected at the quantum level through the tunneling effect. See the right-hand figure of Fig. 2. In [7], a new hilltop inflation scenario has been studied by using the Euclidean time solution (instanton) for this region.

(c) For $C \geq \frac{16 \tilde{\alpha}^{2} \Lambda_{4}}{3} k^{2}\left(1-4 \tilde{\alpha}^{2} \frac{\Lambda_{4}}{3}\right)$, there is no potential barrier and the classical inflationary solution is obtained for $a_{0}^{\min }<a_{0}$.

In the following sections, we consider the time evolution of the Universe in the small $a_{0}$ region by using quantum cosmological approaches. 


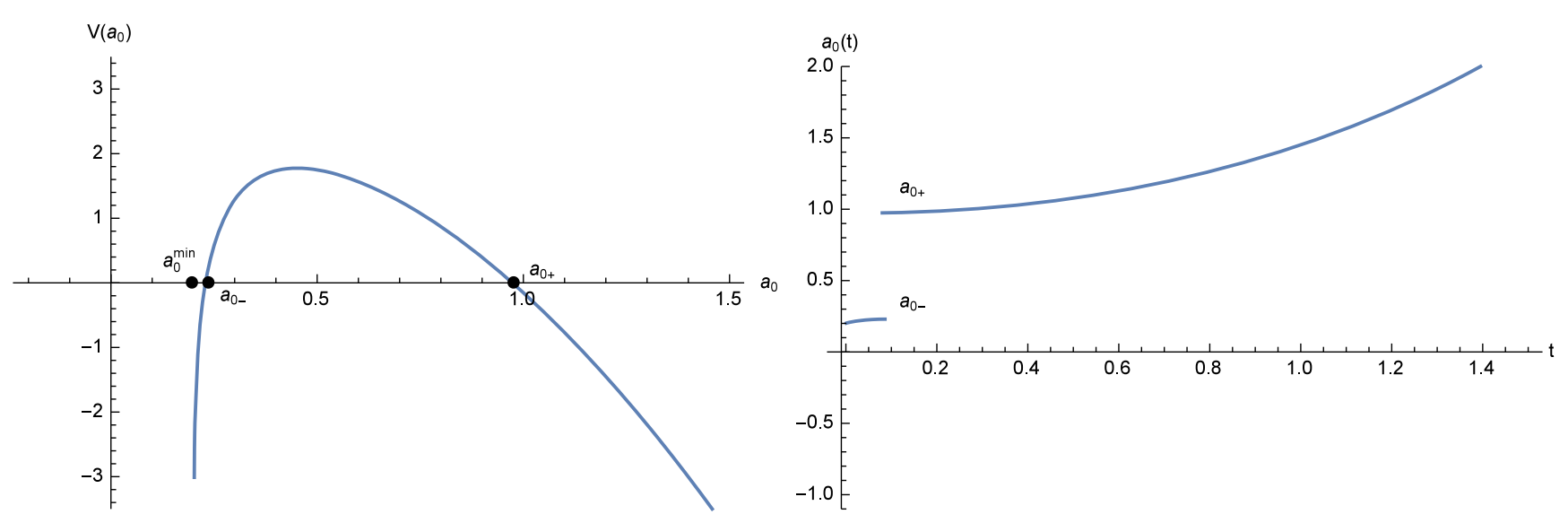

FIG. 2. Left: Potential barrier of $C=1.0$. Right: Two independent classical solutions for $a_{0}(t)$.

\section{QUANTUM COSMOLOGY AND EFFECTIVE ACTION}

There are two quantum mechanical ways to study the small $a_{0}$ region, canonical approach, and path-integral approach. In any case, we need an effective action that leads to the classical equations of motion (2.2) for an appropriate coordinate. It would be impossible to find a general coordinate invariant form since $\left\langle T_{\mu \nu}^{\mathrm{SYM}}\right\rangle$ comes from the conformal anomaly for the CFT.

However, we could find an effective action that provides the classical equations of motion, (2.5) and (2.6), written in the FRW metric. Namely, when the dynamical variable is restricted to the scale factor with a lapse function as a multiplier, it becomes possible to extend the analysis to the quantum cosmology.

Then we restrict ourselves in minisuperspace. Let us consider the metric

$$
d s_{(4)}^{2}=-\mathcal{N}^{2}(t) d t^{2}+a_{0}(t)^{2} \gamma_{i j} d x^{i} d x^{j},
$$

where $\mathcal{N}(t)$ denotes the lapse function. According to the idea of $[4,8]$, the effective action is written as follows ${ }^{3}$ :

$$
\begin{aligned}
S & =\int d^{4} x \sqrt{-g}\left\{\frac{1}{2 \kappa_{4}^{2}}\left(R_{4}-2 \Lambda_{4}\right)+L_{\mathrm{SYM}}^{\mathrm{eff}}\right\} \\
& =V_{3} \int d t \mathcal{N} a_{0}^{3}\left\{\frac{1}{\kappa_{4}^{2}}\left(\frac{3}{a_{0}^{2}}\left(-\frac{\dot{a}_{0}^{2}}{\mathcal{N}^{2}}+k\right)-\Lambda_{4}\right)+L_{\mathrm{SYM}}^{\mathrm{eff}}\right\},
\end{aligned}
$$

where

$$
L_{\mathrm{SYM}}^{\mathrm{eff}}=-\frac{3 N^{2}}{32 \pi^{2}}\left(\frac{4 C}{a_{0}^{4}}+\frac{k^{2}}{a_{0}^{4}}-\frac{2 k}{a_{0}^{2}} \frac{\dot{a}_{0}^{2}}{\mathcal{N}^{2} a_{0}^{2}}-\frac{1}{3 \mathcal{N}^{4}} \frac{\dot{a}_{0}^{4}}{a_{0}^{4}}\right) .
$$

\footnotetext{
${ }^{3}$ Here an appropriate boundary term is abbreviated.
}

The Lagrangian $L_{\mathrm{SYM}}^{\mathrm{eff}}$ is determined such that we could find the Friedmann equation (2.5) from the stationary condition for $\mathcal{N}$ with $\mathcal{N}=1$ gauge. We should notice that (2.6) is also found from the variational equation of $a_{0}$. In this sense, (3.2) with (3.3) leads to a correct form of equations of motion to obtain our classical solutions. It is however difficult to develop an effective quantum theory based upon the action (3.2) due to the term (3.3). The situation is similar to the case with higher curvature terms.

Then we consider an alternative effective action that leads to (2.14) instead of (2.5). It is given as

$$
\begin{aligned}
S & =\int d^{4} x \sqrt{-g} \frac{1}{2 \kappa_{4}^{2}}\left(R_{4}-2 \Lambda_{\mathrm{eff}}\right) \\
& =V_{3} \int d t \mathcal{N} a_{0}^{3} \frac{1}{\kappa_{4}^{2}}\left(\frac{3}{a_{0}^{2}}\left(-\frac{\dot{a}_{0}^{2}}{\mathcal{N}^{2}}+k\right)-\Lambda_{\mathrm{eff}}\right),
\end{aligned}
$$

where

$$
\Lambda_{\text {eff }}^{ \pm}=3 \lambda_{ \pm} \equiv 3 \frac{1 \pm \sqrt{1-4 \tilde{\alpha}^{2}\left(\frac{\Lambda_{4}}{3}+\tilde{\alpha}^{2} \frac{4 C}{a_{0}^{4}}\right)}}{2 \tilde{\alpha}^{2}} .
$$

This action is useful to perform the canonical formulation. In fact, from this action, it is easy to obtain the WDW equation (see Appendix A), which is not written here since we change the variable from $a_{0}$ to $q=a_{0}^{2}$.

\section{A. Change of variables}

According to $[14,15], a_{0}$ and $\mathcal{N}$ are changed as $q=a_{0}^{2}$ and $\mathcal{N} \rightarrow \mathcal{N} / a_{0}$; then we have

$$
\begin{gathered}
S=\frac{V_{3}}{\kappa_{4}^{2}} \int d t\left(-\frac{3 \dot{q}^{2}}{4 \mathcal{N}}+\mathcal{N}\left(3 k-q \Lambda_{\text {eff }}\right)\right), \\
\Lambda_{\text {eff }}=3 \frac{1-\sqrt{1-4 \tilde{\alpha}^{2}\left(\frac{\Lambda_{4}}{3}+\tilde{\alpha}^{2} \frac{4 C}{q^{2}}\right)}}{2 \tilde{\alpha}^{2}} .
\end{gathered}
$$




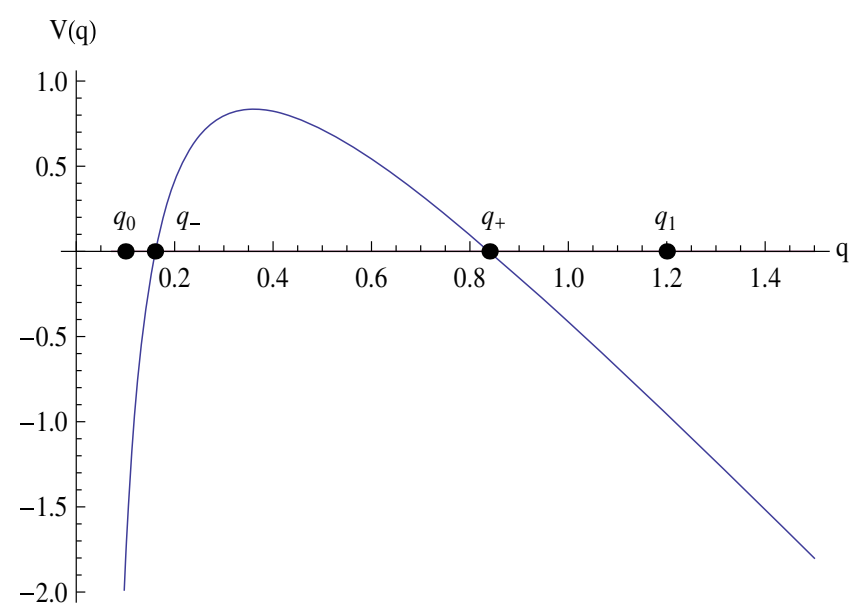

FIG. 3. Plots of $V_{\text {eff }}(q)$ vs $q$ for $\Lambda_{4}=3, k=1, \tilde{\alpha}^{2}=0.01$ and $C=31.3$.

In this formulation, the WDW equation is given as

$$
\left(-\hbar^{2} \frac{\partial^{2}}{\partial q^{2}}+V(q)\right) \Psi(q)=0
$$

which is also obtained by changing the variable as $q=a_{0}^{2}$ in (A12). The potential $V(q)$ is given by

$$
V_{\mathrm{eff}}(q) \equiv V(q) /\left(3 v_{3}^{2}\right)=3 k-q \Lambda_{\mathrm{eff}}(q)
$$

\section{B. $V_{\text {eff }}(q)$ and tunneling}

As shown in Sec. II, we find similar behavior of the potential $V_{\text {eff }}(q)$ to the case of $V\left(a_{0}\right)$. The typical potentials are similar to Fig. 1, so they are abbreviated here.

Here we should notice as mentioned in Sec. II that there is a lower bound of $q$ for $C>0$. It corresponds to $a_{0}^{\text {nin }}$ given in the previous section. The bound is given as

$$
q^{\min }=\tilde{\alpha}^{2}\left(\frac{16 C}{\tilde{\Lambda}_{4}}\right)^{1 / 2}, \quad \tilde{\Lambda}_{4}=1-\frac{4 \tilde{\alpha}^{2} \Lambda_{4}}{3} .
$$

For $q<q^{\min }$, the potential becomes complex. So we cannot extend our model in this region. Therefore we concentrate our analysis on the region $q>q^{\mathrm{min}}$.

In this allowed region, several types of potentials are seen depending on the value of $C$. Hereafter we study the case shown in Fig. 3. This has two turning points, say $q_{-}$, and $q_{+}\left(>q_{-}\right)$, in the two classical regions. For very small $C$, small sized universes may be made but they soon disappear into the region of $q<q^{\min }$ where EinsteinHilbert action is not available. However some of them go through the mountain of the potential via a quantum tunneling effect. From the viewpoint of the inflational scenario, this quantum jump of a small sized universe gives us a clue to the initial condition of the inflation.
Then, by using this potential, the tunneling birth of our Universe can be studied.

In principle, it is possible to calculate the propagator, $G\left(q_{1}, q_{0}\right)$, where the points $q_{0}$ and $q_{1}$ are shown in Fig. 3, according to the path integral as discussed above in order to see the tunneling effect. However, it is difficult to find a saddle point in the complex $\mathcal{N}$ plane in the present model. So we perform the same calculation in terms of solving the WDW equation given as follows.

\section{WDW EQUATION AND TUNNELING}

Considering the potential as shown in Fig. 3, we give the tunneling amplitude for $C>0$ by solving the WDW equation (3.8). Supposing the form of the wave function $\Psi(q)$ is as follows,

$$
\Psi(q)=A(q) e^{i \phi(q) / \hbar},
$$

the WDW equation (3.8) leads to the following equations:

$$
\begin{gathered}
\left(\partial_{q} \phi\right)^{2}+12 \pi^{4} V_{\mathrm{eff}}=\frac{\hbar^{2}}{A} \partial_{q}^{2} A, \\
\partial_{q} A \partial_{q} \phi+\frac{1}{2} A \partial_{q}^{2} \phi=0 .
\end{gathered}
$$

They are solved by expanding $\phi$ with the power of $\hbar$.

In the regions of $q<q_{-}$and $q_{+}<q$, the wave functions $\Psi_{1}$ and $\Psi_{3}$ are obtained in the following forms,

$$
\begin{aligned}
& \Psi_{1}=\frac{c_{+}}{\sqrt{k(q)}} e^{-i \eta\left(q, q_{-}\right)}+\frac{c_{-}}{\sqrt{k(q)}} e^{i \eta\left(q, q_{-}\right)}, \\
& \Psi_{3}=\frac{D_{+}}{\sqrt{k(q)}} e^{-i \eta\left(q_{+}, q\right)}+\frac{D_{-}}{\sqrt{k(q)}} e^{i \eta\left(q_{+}, q\right)},
\end{aligned}
$$

where both the first terms of $\Psi_{1}$ and $\Psi_{3}$ represent the outgoing (growing) wave, and

$$
k(q)=\sqrt{|V|} / \hbar, \quad \eta\left(q, q_{-}\right)=\int_{q}^{q_{-}} d q^{\prime} k\left(q^{\prime}\right),
$$

where we notice that $\eta\left(q, q_{-}\right)=P\left(q, q_{-}\right)$, which is defined in the next section to express the Green function $G\left(q, q_{-}\right)$.

We can set various boundary conditions for the solutions of the wave function $\Psi_{q}$. At first, we concentrate on the tunneling. In order to see the tunneling amplitude, we impose the condition $D_{-}=0$. Namely, only the outgoing wave is restricted in the region $q_{+}<q$; then we find

$$
D_{+}=c_{+} /\left(\frac{1}{4} e^{-\eta\left(q_{-}, q_{+}\right)}+e^{\eta\left(q_{-}, q_{+}\right)}\right) .
$$

Then we find the tunneling probability 


$$
T \simeq\left|\frac{D_{+}}{c_{+}}\right|^{2}=e^{-2 P\left(q_{+}, q_{-}\right)} .
$$

We expect that this result could also be found from the propagator given by the path integral discussed above [14]. In order to understand the role of $C$, it would be useful to show the $C$-dependence of the probability $T$. It is shown in the Fig. 4 for three values of $\Lambda_{4}$.

On the other hand, for the condition $D_{+}=-i D_{-}$, we find the relation

$$
c_{+}=\frac{D_{+}}{2} e^{-\eta\left(q_{-}, q_{+}\right)}
$$

This leads to

$$
\left|\frac{D_{+}}{c_{+}}\right|^{2}=4 e^{2 P\left(q_{+}, q_{-}\right)} .
$$

The sign of the exponent of $\left|\frac{D_{+}}{c_{+}}\right|^{2}$ in this case is opposite to the tunneling case. This result corresponds to the one obtained in [17] as the wave function of the WDW equation.

There are many other conditions, which lead to various forms of $\left|\frac{D_{+}}{c_{+}}\right|^{2}$. The point we want to see is how these solutions of the WDW equation are related to the results of the path integral. The saddle points given from the effective action of complex $\mathcal{N}$ can be related to the above solutions of the WDW equation. In order to answer this question, we consider a simple model that has the properties of the above holographic model for the $C>0$ case.

\section{QUANTUM COSMOLOGY WITH LORENTZIAN PATH INTEGRAL}

In the previous section, we considered canonical formalism to find the wave function of the Universe by the WDW equation. In this section, let us consider the pathintegral formalism to get the propagator of the Universe.

The Feynman propagator in minisuperspace is defined as [14]

$$
G\left(q_{1}, q_{0}\right)=\int_{0^{+}}^{\infty} d \mathcal{N} \int_{q_{0}}^{q_{1}} D q e^{i S(\mathcal{N}, q) / \hbar},
$$

where $q_{0}=q(0)$ and $q_{1}=q\left(t_{1}\right)$ are the initial and final values of $q(t)$. After integrating over $q$, we are left with the integration over $\mathcal{N}$. In order to perform the integration, we here try to apply the Lefschetz thimble method. In Appendix C, the detail of this method is shown.

In this method, the original path is extended to the complex plane,

$$
\mathcal{N}=u_{1}+i u_{2},
$$

where both $u_{1}=\operatorname{Re}(\mathcal{N})$ and $u_{2}=\operatorname{Im}(\mathcal{N})$ are real, and the propagator is evaluated by the saddle-point approximation in the $\hbar \rightarrow 0$ limit. Below we see those examples concretely.

\section{A. The case of $C=0$}

In this case, the action (3.4) is equivalent to the one with the Einstein-Hilbert term and a cosmological constant. So, it has been already studied in [14]. Equation of motion for $q(t)$ and the constraint from (3.6) are

$$
\begin{gathered}
\ddot{q}=\frac{2}{3} \mathcal{N}^{2} \Lambda_{\text {eff }}, \\
\frac{3 \dot{q}^{2}}{4 \mathcal{N}^{2}}+3 k=q \Lambda_{\text {eff }} .
\end{gathered}
$$

The path integral over $q$ becomes Gaussian and is exactly treated. Then we are left with the integral over $\mathcal{N}$ as follows:

$$
G\left(q_{1}, q_{0}\right)=\sqrt{\frac{3 \pi i}{2 \hbar}} \int_{0}^{\infty} \frac{d \mathcal{N}}{\mathcal{N}^{1 / 2}} e^{2 \pi^{2} i S(\mathcal{N})},
$$

where

$S(\mathcal{N})=\frac{1}{36} \mathcal{N}^{2} \Lambda_{\text {eff }}^{2}+\left(3 k-\frac{1}{2}\left(q_{0}+q_{1}\right)\right) \mathcal{N}-\frac{3}{4 \mathcal{N}}\left(q_{1}-q_{0}\right)^{2}$

The action (5.6) has four saddle points in the complex $\mathcal{N}$ plane. If we choose, for instance, $k=1, \Lambda_{\text {eff }}=3, q_{0}=0$, and $q_{1}=10$, those points lie at $(3, i),(3,-i),(-3, i)$, and $(-3,-i)$. Then, by using the thimble decomposition (as described in Appendix C), it is found that the original contour is deformed to the Lefschetz thimble $J_{1}$ so that only one saddle $\mathcal{N}_{1}=(3, i)$ contributes to the integral (see Fig. 5).

Here we notice that the authors of Ref. [14] have shown that the tunneling probability from $q_{0}$ to $q_{1}$ is precisely obtained by the propagator $G\left(q_{1}, q_{0}\right)$, which could reproduce the correct boundary condition of the tunneling.

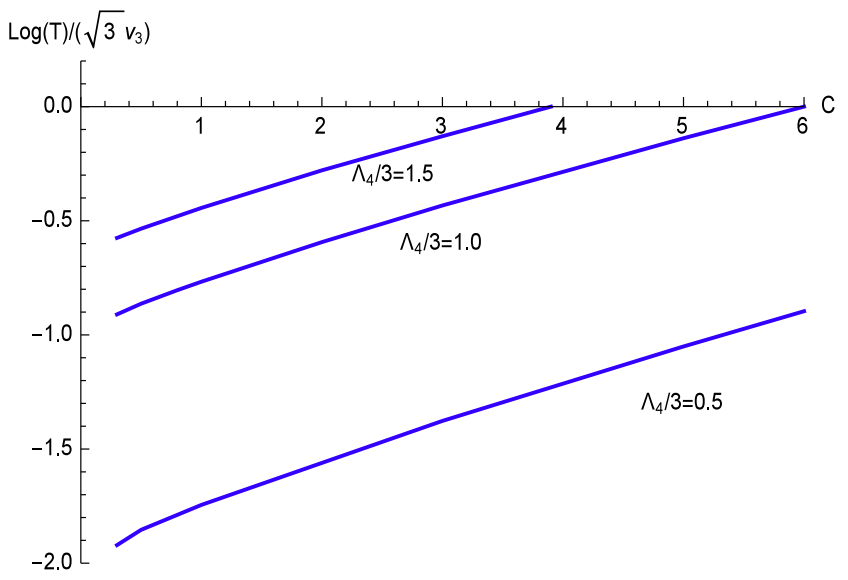

FIG. 4. The $C$ dependence of the tunneling probability $T$ for different values of $\Lambda_{4}$. 


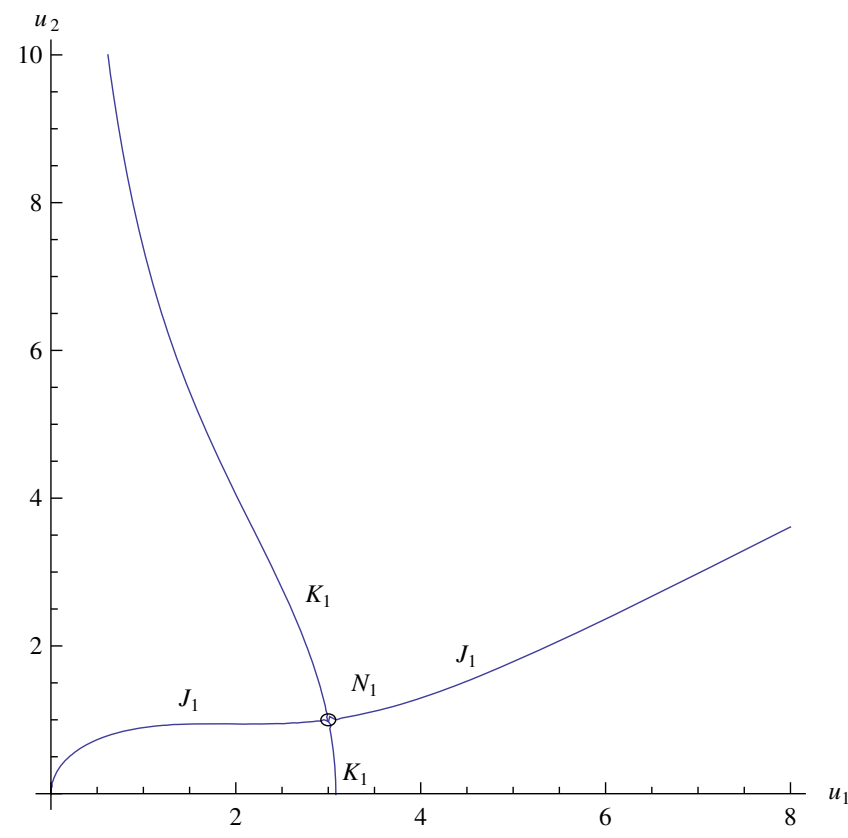

FIG. 5. Plots of Lefschetz thimble $J_{1}$ (the steepest descent) and $K_{1}$ (the steepest ascent) for the saddle $\mathcal{N}_{1}=(3, i)$, where $\Lambda_{\text {eff }}=3, k=1, q_{0}=0, q_{1}=10$.

According to this observation, the propagator obtained in this way is interpreted as the tunneling probability factor when the initial value $q_{0}$ is in the quantum region and the final point is at the zero point of $V_{\text {eff }}$. It is given by

$$
G\left(q_{1}, q_{0}\right) \propto e^{-2 P\left(q_{1}, q_{0}\right)},
$$

where

$$
\begin{aligned}
P\left(q_{1}, q_{0}\right) & =\frac{v_{3}}{\hbar} \int_{q_{0}}^{q_{1}} \sqrt{3 V_{\mathrm{eff}}^{(0)}(q)} d q, \\
V_{\mathrm{eff}}^{(0)}(q) & =3 k-q \Lambda_{\mathrm{eff}}^{(C=0)}(q) .
\end{aligned}
$$

Here the oscillating part is abbreviated. This term precisely denotes the tunneling probability found in the WentzelKramers-Brillouin approximation of the WDW equation in the previous section.

Here is a comment. In this case, we find another three saddle points that do not contribute to the integration. However, they might affect in some case where the integration path is defined in a different way [17].

The tunneling factor (5.8) appears whenever either $q_{0}$ or $q_{1}$ is in the quantum region. So the Lefschetz thimble method is useful to study quantum cosmology and it is equivalent to solve the WDW equation under appropriate boundary conditions.

\section{B. The case of $C \neq 0$}

It is difficult to perform the path integral for the potential $V_{\text {eff }}(q)$ with $C \neq 0$ due to a complicated $q$ dependence of $V_{\text {eff }}(q)$. Then let us consider the following action, that is,

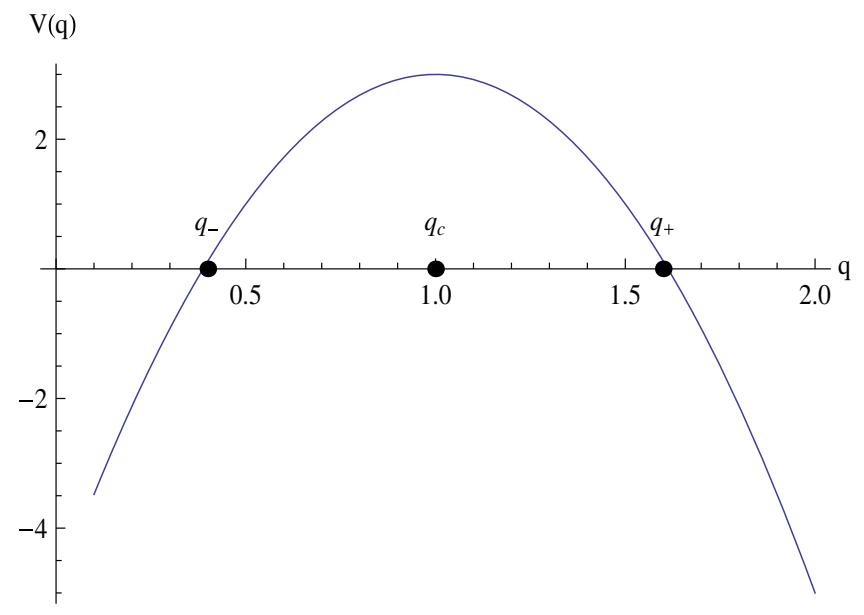

FIG. 6. Plots of $V_{\text {sim }}=V(q)$, the simplified model (5.10), for $q_{c}=1, \mu=8$, and $k=1 . q_{ \pm}$denote the zero points of $V(q)$.

$$
S=v_{3} \int d t\left(-\frac{3 \dot{q}^{2}}{4 \mathcal{N}}+\mathcal{N}\left(3 k-\mu\left(q_{c}-q\right)^{2}-\beta\right)\right) \quad(\mu>0)
$$

Here the original $V_{\text {eff }}(q)$ defined by (3.9) is approximated by a quadratic form near its maximum point, $q_{c}$.

By doing this, one can study the model analytically without losing characteristic properties of the original model within the approximation mentioned above. Then we use the following potential $V_{\text {sim }}$ (see Fig. 6),

$$
V_{\text {sim }} \equiv 3 k-\mu\left(q_{c}-q\right)^{2}-\beta,
$$

where $\mu$ and $\beta$ are constant parameters. Hereafter we discuss the case of $\beta=0$.

Equation of motion for $q$ is easily solved as

$$
q=q_{c}+a_{+} e^{\frac{\mathcal{N}}{t_{0}} t}+a_{-} e^{-\frac{\mathcal{N}}{t_{0}} t}, \quad t_{0} \equiv \sqrt{\frac{3}{4 \mu}} .
$$

The coefficients $a_{ \pm}$are determined through the boundary conditions, $q(0)=q_{0}$ and $q(1)=q_{1}$,

$$
\begin{gathered}
a_{+}=-\frac{\tilde{q}_{0} e^{-\frac{\mathcal{N}}{t_{0}}}-\tilde{q}_{1}}{2 \sinh \left(\frac{\mathcal{N}}{t_{0}}\right)}, \\
a_{-}=\frac{\tilde{q}_{0} e^{\frac{\mathcal{N}}{t_{0}}}-\tilde{q}_{1}}{2 \sinh \left(\frac{\mathcal{N}}{t_{0}}\right)}, \\
\tilde{q}_{i} \equiv q_{i}-q_{c} .
\end{gathered}
$$

By substituting these coefficients into (5.9), we get 
$S_{\mathrm{sim}}^{(0)}=v_{3} \mu t_{0}\left(\frac{3 k \mathcal{N}}{\mu t_{0}}-\frac{\left(\tilde{q}_{1}^{2}+\tilde{q}_{0}^{2}\right) \cosh \left(\frac{\mathcal{N}}{t_{0}}\right)-2 \tilde{q}_{1} \tilde{q}_{0}}{\sinh \left(\frac{\mathcal{N}}{t_{0}}\right)}\right)$.

From the stationary condition $\delta S_{\text {sim }}^{(0)} / \delta \mathcal{N}=0$, we find two saddle points, say $\mathcal{N}_{ \pm}$,

$\mathcal{N}_{ \pm}=t_{0} \cosh ^{-1}\left\{\frac{\mu}{3 k}\left(\tilde{q}_{0} \tilde{q}_{1} \pm \sqrt{\left(\tilde{q}_{0}^{2}-\frac{3 k}{\mu}\right)\left(\tilde{q}_{1}^{2}-\frac{3 k}{\mu}\right)}\right)\right\}$.

In order to solve (5.16), we parametrize $\mathcal{N}$ in the polar coordinate $(r, \theta)$,

$$
e^{\frac{\mathcal{N}}{t_{0}}}=r e^{i \theta} \quad \text { or } \quad \mathcal{N}=(\log r+i \theta) t_{0} .
$$

Then (5.16) can be rewritten in terms of $r$ and $\theta$,

$$
\begin{aligned}
\cosh \left(\frac{\mathcal{N}}{t_{0}}\right) & =\frac{1}{2}\left[\left(r+\frac{1}{r}\right) \cos \theta+i\left(r-\frac{1}{r}\right) \sin \theta\right] \\
& =Q_{0} Q_{1} \pm \sqrt{\left(Q_{0}^{2}-1\right)\left(Q_{1}^{2}-1\right)} \equiv X_{ \pm},
\end{aligned}
$$

where

$Q_{0}=\frac{\tilde{q}_{0}}{\left|\tilde{q}_{ \pm}\right|}, \quad Q_{1}=\frac{\tilde{q}_{1}}{\left|\tilde{q}_{ \pm}\right|}, \quad \tilde{q}_{ \pm}= \pm \sqrt{\frac{3 k}{\mu}}$.

Note that $\tilde{q}_{ \pm}$denote the 0's of the potential term $V_{\text {sim }}$ defined by (5.10).

\section{Tunneling}

Let us consider a situation in which $q_{0}$ and $q_{1}$ are put on the opposite side of the hill of the potential (see Fig. 7). The

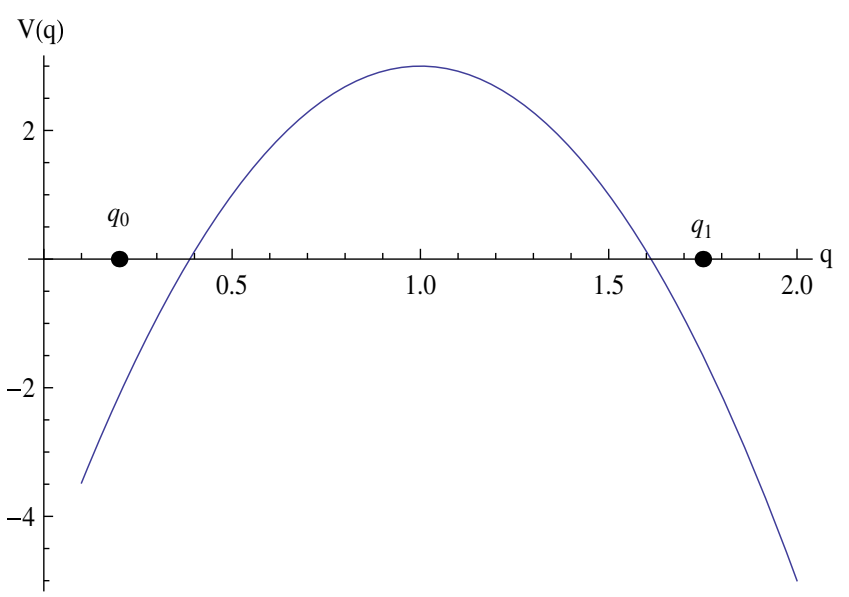

FIG. 7. Plots of the typical position of $q_{0}$ and $q_{1}$ for the tunneling process are shown with $V_{\text {sim }}=V(q)$, the simplified model (5.10) shown in Fig. 6. transition from $q_{0}$ to $q_{1}$ is realized by the quantum tunneling.

In this case, since $Q_{0}<-1$ and $Q_{1}>1, X_{ \pm}$in (5.18) are real and satisfy an inequality $X_{-}<X_{+}<-1$. Then we obtain

$$
\begin{array}{r}
X_{ \pm}=-\frac{1}{2}\left(r_{ \pm}+\frac{1}{r_{ \pm}}\right) \quad(<-1), \\
\theta= \pm(2 n+1) \pi, \quad n=0, \pm 1, \pm 2, \ldots
\end{array}
$$

From this, we find two values of $r$, at $r_{ \pm l}>1$ and $r_{ \pm s}<1$, for $X_{+}$. So there are eight saddle points for one $|\theta|$ in the complex $\mathcal{N}$ plane. In Fig. 8, saddle points for $n=0$ with some specific parameters are shown.

We should notice that there are no real $\mathcal{N}$ solutions in the present case. This implies that the path connecting $q_{0}$ and $q_{1}$ represents a quantum process.

For the saddle points with $\theta=\pi(n=0)$ and $r=r_{ \pm s}$, the action is evaluated as

$$
\begin{aligned}
\operatorname{Re}\left[\frac{2 \pi^{2}}{\hbar} i S_{0}\right] & =-\frac{2 \pi^{2}}{\hbar} 3 \pi t_{0}=-\frac{3 \sqrt{3} \pi^{3}}{\sqrt{\mu} \hbar} \\
& =-\frac{\sqrt{12} \pi^{2}}{\hbar} \int_{\tilde{q}_{-}}^{\tilde{q}_{+}} \sqrt{3 k-\mu \tilde{q}^{2}} d \tilde{q}=-P\left(\tilde{q}_{+}, \tilde{q}_{-}\right) .
\end{aligned}
$$

Then the factor $e^{-P\left(\tilde{q}_{+}, \tilde{q}_{-}\right)}$corresponds to the tunneling amplitude.

As for the above solution, the propagator has a slightly different phase from the one of $r=r_{ \pm s}$. This implies that this solution corresponds to the propagator traveling a slightly long path in the classical region $0<q<q_{-}$.

The propagator is calculated by integrating the rhs of (5.1) over $q$, and the integration path of $\mathcal{N}$ is deformed to a curve in the first quadrant of the complex $\mathcal{N}$ plane. In the

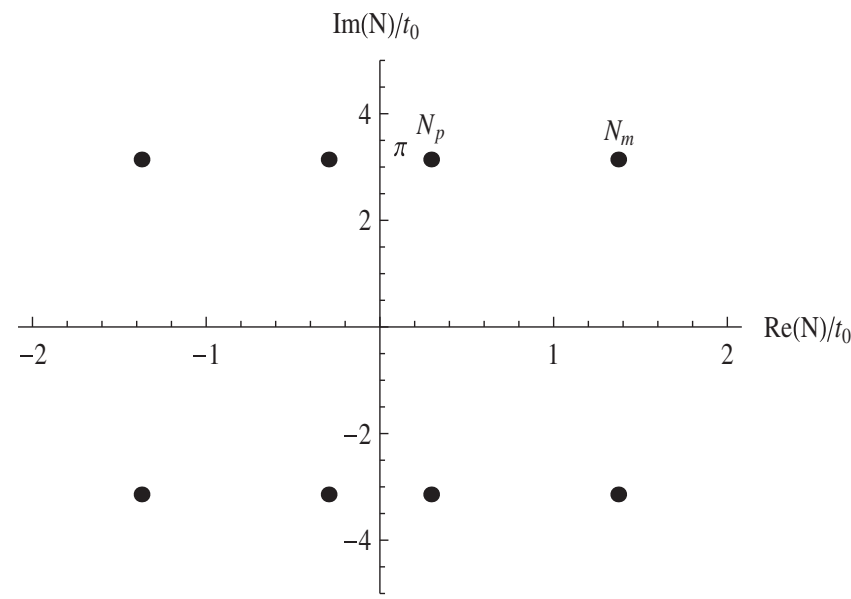

FIG. 8. Plots of the saddle point of $\mathcal{N}$ for the simplified model. Here the case of $\theta=\pi, \tilde{q}_{0}=-\tilde{q}_{+}(1+0.2)$, and $\tilde{q}_{1}=\tilde{q}_{+}(1+0.5)$ is shown. 




FIG. 9. A sketch of the thimbles: Because of the degeneracy between steepest descent and ascent flows (thimbles and dual thimbles), it is not possible to classify the flow segments, definitely. For each saddle point on the relevant integration path, a neighboring segment of a steepest descent flow is labeled with $J_{i}$ and one of a steepest ascent flow is labeled with $K_{i}$.

method of the Lefschetz thimble, the curved path contains some steepest descent flows of $\operatorname{Re}\left[i S_{\min }^{(0)}(\mathcal{N})\right]$. As shown in Fig. 9, there are two possible saddle points each of which connects to the origin $\mathcal{N}=0$ through a steepest descent flow (a Lefschetz thimble). One is $\mathcal{N}_{p}^{(n=0)}=$ $\left(-\cosh ^{-1}\left(-Q_{0}\right)+\cosh ^{-1} Q_{1}+i \pi\right) t_{0}$ in the $\mathcal{N}_{p}$ series and another is $\mathcal{N}_{m}^{(n=0)}=\left(\cosh ^{-1}\left(-Q_{0}\right)+\cosh ^{-1} Q_{1}+i \pi\right) t_{0}$ in the $\mathcal{N}_{m}$ series.

The thimble that passes the former saddle point $\mathcal{N}_{p}^{(n=0)}$ connects to the singular point at $\mathcal{N}=i(\pi+0) t_{0}$, and is terminated there. This means that the thimble attached to this saddle point is irrelevant to the present method. On the other hand, the thimble that passes the latter saddle point
$\mathcal{N}_{m}^{(n=0)}$ does not reach any singular point before the next saddle point $\mathcal{N}_{m}^{(n=1)}=\left(\cosh ^{-1}\left(-Q_{0}\right)+\cosh ^{-1} Q_{1}+3 i \pi\right) t_{0}$. This thimble is shown as the set of $J_{1}^{L}$ and $J_{1}^{R}$ in Fig. 9 and also in the left panel of Fig. 10. Furthermore, its dual thimble (the steepest ascent flow) intersects the real axis of the complex $\mathcal{N}$ plane. Therefore, the relevant path should run from the origin toward the saddle point $\mathcal{N}_{m}^{(n=0)}$. Now, the Lefschetz thimbles are obtained as the flow lines emanating from the saddle points of the $\mathcal{N}_{m}$ series, as shown in the left of Fig. 10.

However, the situation is somewhat complicated because of the degeneracy between the flow lines $J_{i}$ and $K_{i+1} \cdot{ }^{4}$ To find the appropriate path over the saddle point $\mathcal{N}_{m}^{(n=1)}$, one may try to provide a perturbation term to the original action (5.15), for example, $\Delta S_{0}=i \mathcal{N} / 100$. Actually, $\Delta S_{0}$ removes the degeneracy as depicted by the right panel of Fig. 10. Finally, setting $\Delta S_{0}=0$ again, one finds a unique path as $J_{1}^{L} \rightarrow J_{1}^{R} \rightarrow K_{2}^{R} \rightarrow J_{2}^{R} \rightarrow \cdots$, where only the first saddle point $\mathcal{N}_{m}^{(n=0)}$ dominates the integration.

Tunneling probability via steepest descent method.-The tunneling probability is estimated by using the propagator (5.1), and in the simplified model the amplitude is reduced to

$$
G\left(q_{1}, q_{0}\right)=\int_{0^{+}}^{\infty} \sqrt{\frac{3 i / 2 t_{0}}{2 \pi \sinh \left(\frac{\mathcal{N}}{t_{0}}\right)}} e^{i S_{\text {sim }}^{(0)}(\mathcal{N})} d \mathcal{N}
$$

after integrating over $q$.

As mentioned above, $G\left(q_{1}, q_{0}\right)$ is approximately evaluated by the main contribution of the saddle point at

$$
\mathcal{N}_{0} \equiv \mathcal{N}_{m}^{(n=0)}=\left(\cosh ^{-1}\left(-Q_{0}\right)+\cosh ^{-1} Q_{1}+i \pi\right) t_{0},
$$

which is the joint of $J_{1}^{L}$ and $J_{1}^{R}$. Then, the amplitude (5.23) is calculated as follows:

$$
\begin{aligned}
G\left(q_{1}, q_{0}\right) & \simeq \sqrt{\frac{3 i / 2 t_{0}}{2 \pi \sinh \left(\frac{\mathcal{N}}{t_{0}}\right)}} e^{i \operatorname{Re} S_{\text {sim }}^{(0)}\left(\mathcal{N}_{0}\right)} \int_{J_{1}^{L}+J_{1}^{R}} e^{-\operatorname{Im} S_{\mathrm{sim}}^{(0)}(\mathcal{N})} d \mathcal{N} \\
& \simeq \sqrt{\frac{3 i / 2 t_{0}}{2 \pi \sinh \left(\frac{\mathcal{N}}{t_{0}}\right)}} e^{i \operatorname{Re} S_{\mathrm{sim}}^{(0)}\left(\mathcal{N}_{0}\right)} \int_{-\infty}^{\infty} e^{-\operatorname{Im} S_{\mathrm{sim}}^{(0)}\left(\mathcal{N}_{0}\right)-\frac{1}{2}\left|S_{\mathrm{sim}}^{(0) \prime \prime}\left(\mathcal{N}_{0}\right)\right| \nu^{2}} e^{i \theta_{0}} d \nu \\
& =\sqrt{\frac{3 i / 2 t_{0}}{2 \pi \sinh \left(\frac{\mathcal{N}}{t_{0}}\right)}} e^{i\left(\operatorname{Re} S_{\mathrm{sim}}^{(0)}\left(\mathcal{N}_{0}\right)+\theta_{0}\right)} e^{-\operatorname{Im} S_{\mathrm{sim}}^{(0)}\left(\mathcal{N}_{0}\right)} \sqrt{\frac{2 \pi}{\left|S_{\mathrm{sim}}^{(0) \prime \prime}\left(\mathcal{N}_{0}\right)\right|}}
\end{aligned}
$$

\footnotetext{
${ }^{4}$ Here we consider that the suffix is identified with $n=i$.
} 

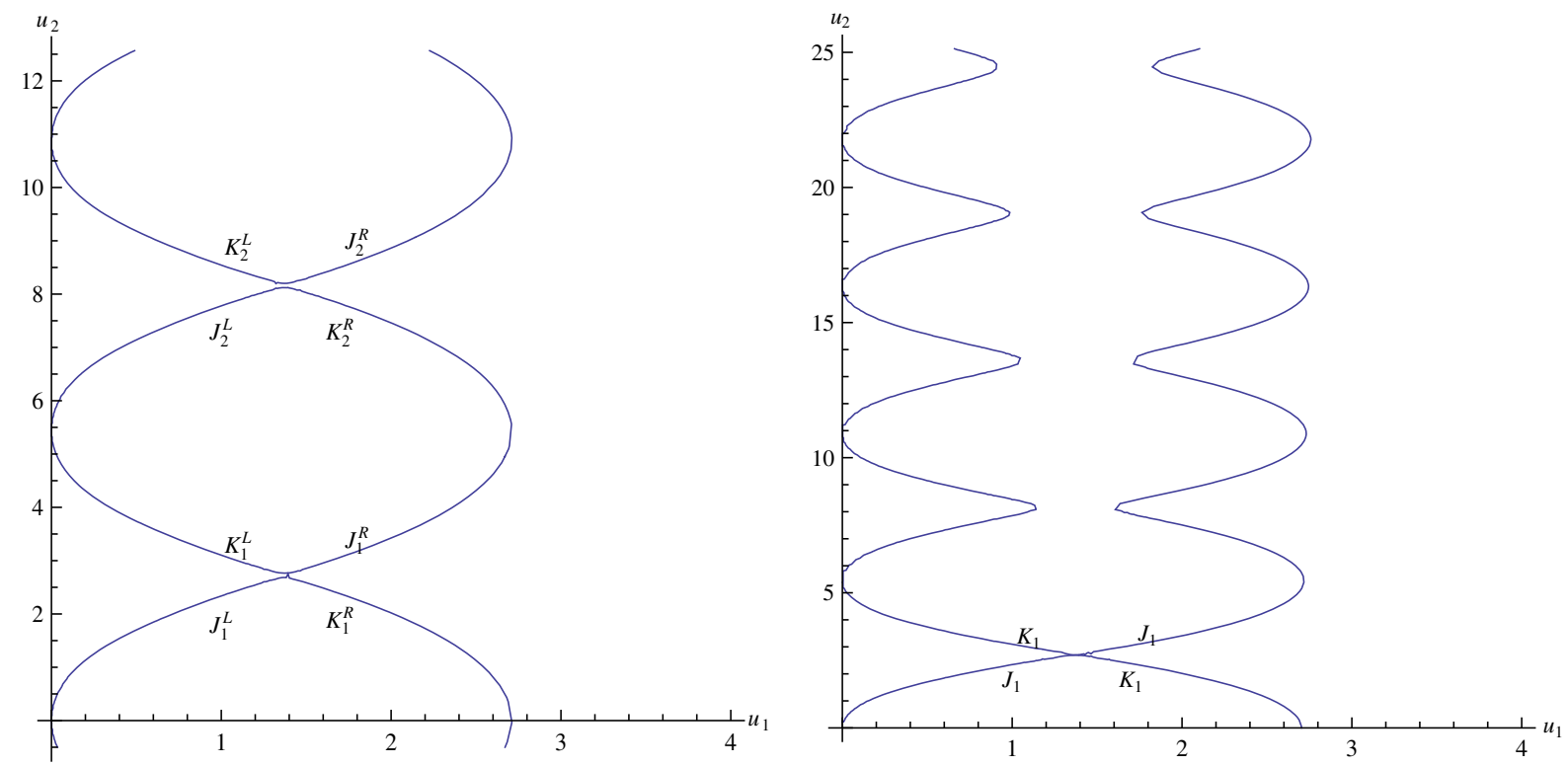

FIG. 10. Left: Plots of the Lefschetz thimbles for $\mathcal{N}_{m}$ of the simplified model. Here the case of $\theta=\pi, \tilde{q}_{0}=-\tilde{q}_{+}(1+0.2)$, and $\tilde{q}_{1}=\tilde{q}_{+}(1+0.5)$ is shown. Right: The thimbles corresponding to the first saddle of $\mathcal{N}_{m}$ series or an integral path when a perturbation $\Delta S_{0}=i \mathcal{N} / 100$ is included.

where $\theta_{0}$ is the incident angle of the thimble $J_{1}^{L}$ into the point $\mathcal{N}_{0}$ in the complex $\mathcal{N}$ plane. The angle $\theta_{0}$ is determined by

$$
\tan \theta_{0}=\frac{\operatorname{Re} S_{\text {sim }}^{(0) \prime}\left(\mathcal{N}_{0}\right)}{\left|S_{\text {sim }}^{(0) \prime}\left(\mathcal{N}_{0}\right)\right|+\operatorname{Im} S_{\text {sim }}^{(0) "}\left(\mathcal{N}_{0}\right)}=\frac{\left|S_{\text {sim }}^{(0) \prime \prime}\left(\mathcal{N}_{0}\right)\right|-\operatorname{Im} S_{\text {sim }}^{(0) \prime}\left(\mathcal{N}_{0}\right)}{\operatorname{Re} S_{\text {sim }}^{(0) "}\left(\mathcal{N}_{0}\right)} .
$$

Since it holds that

$$
S_{\text {sim }}^{(0) "}(\mathcal{N})=\frac{3 v_{3} k}{t_{0}} F^{(2)}\left(\frac{\mathcal{N}}{t_{0}}\right)
$$

with

$$
F^{(2)}\left(\frac{\mathcal{N}}{t_{0}}\right)=\left[\frac{2 Q_{0} Q_{1} \cosh ^{2}\left(\frac{\mathcal{N}}{t_{0}}\right)-2\left(Q_{0}^{2}+Q_{1}^{2}\right) \cosh \left(\frac{\mathcal{N}}{t_{0}}\right)+2 Q_{0} Q_{1}}{\sinh ^{3}\left(\frac{\mathcal{N}}{t_{0}}\right)}\right],
$$

the second order derivative $S_{\text {sim }}^{(0) /}(\mathcal{N})$ satisfies that

$$
\operatorname{Re} S_{\text {sim }}^{(0) \prime}\left(\mathcal{N}_{0}\right)>0, \quad \operatorname{Im} S_{\text {sim }}^{(0) \prime}\left(\mathcal{N}_{0}\right)=0 .
$$

Therefore, the angle $\theta_{0}$ is found to be $\frac{\pi}{4}$.

Now, the absolute value of the tunneling amplitude is given by

$$
\begin{aligned}
\left|G\left(q_{1}, q_{0}\right)\right| & =\sqrt{\frac{3 / 2 t_{0}}{\left|\sinh \left(\frac{\mathcal{N}}{t_{0}}\right) S_{\text {sim }}^{(0) \prime \prime}\left(\mathcal{N}_{0}\right)\right|}} e^{-\operatorname{Im} S_{\text {sim }}^{(0)}\left(\mathcal{N}_{0}\right)} \\
& =\sqrt{\frac{1}{2 v_{3} k\left|\sinh \left(\frac{\mathcal{N}}{t_{0}}\right) F^{(2)}\left(\frac{\mathcal{N}}{t_{0}}\right)\right|}} e^{-\operatorname{Im} S_{\text {sim }}^{(0)}\left(\mathcal{N}_{0}\right),}
\end{aligned}
$$

in which the factor $\left|\sinh \left(\mathcal{N}_{0} / t_{0}\right) F^{(2)}\left(\mathcal{N}_{0} / t_{0}\right)\right|$ is a certain function of $Q_{0}$ and $Q_{1}$, and

$$
-\operatorname{Im} S_{\text {sim }}^{(0)}\left(\mathcal{N}_{0}\right)=-3 k v_{3} t_{0} \pi
$$

We can see $\operatorname{Im} S_{\text {sim }}^{(0)}\left(\mathcal{N}_{0}\right)=P\left(q_{+}, q_{-}\right)$, where $P\left(q_{+}, q_{-}\right)$is defined by (4.6) in Sec. IV when $V$ is replaced by the simplified potential $V_{\text {sim }}$ used in the present section. This implies that the result obtained here reproduces the tunneling probability $e^{-2 P\left(q_{+}, q_{-}\right)}$given in Sec. IV. From Eq. (5.33), it is found that $\left|G\left(q_{1}, q_{0}\right)\right|^{2}$ depends on $t_{0}$ such as

$$
\left|G\left(q_{1}, q_{0}\right)\right|^{2} \propto e^{-6 k v_{3} t_{0} \pi} .
$$




\section{Saddles for periodic Euclidean solution}

In the previous subsection, we considered the saddlepoint contribution from the $n=0$ sector. Then a natural question arises: What is the meaning of other infinite series of the saddle points for $n \geq 1$ ? We could answer the question by considering the "instanton" [7]. In [7], the authors have found a periodic Euclidean solution of the Friedmann equation regarded as an instanton one, which oscillates between $q_{-}$and $q_{+}$.

In the following, let us estimate the instanton contribution by using the Euclidean path-integral formalism. In our simplified model with $V_{\text {sim }}$, equations of motion for $q$ in the Euclidean time $\tau$ are given by

$$
\left(\frac{\partial q}{\partial \tau}\right)^{2}=-\frac{4}{3} \mu\left(q-q_{c}\right)^{2}+4 k
$$

Then the solution is easily obtained as

$q_{I}=\sqrt{\frac{3}{\mu}} \sin \left(\sqrt{\frac{4 \mu}{3}}\right)+q_{0}=2 t_{0} \sin \left(\frac{\tau}{t_{0}}\right)+q_{0}$.

The period of the solution, $T_{0}$, becomes

$$
T_{0}=2 \pi t_{0}=\pi \sqrt{\frac{3}{\mu}}
$$

Then we get the Euclidean action for the instanton solution,

$$
\begin{aligned}
S_{0}^{E} & =i v_{3} \int_{0}^{T_{0}} d \tau\left(\frac{3}{4} \dot{q}^{2}+3 k-\mu\left(q-q_{c}\right)^{2}\right) \\
& =i v_{3} \times 3 T_{0} .
\end{aligned}
$$

Therefore, we find

$\operatorname{Re}\left[\frac{2 \pi^{2}}{\hbar} i S_{0}^{E}\right]=-\frac{2 \pi^{2}}{\hbar} 6 \pi t_{0}=-\frac{6 \sqrt{3} \pi^{3}}{\sqrt{\mu} \hbar}=-2 P\left(\tilde{q}_{+}, \tilde{q}_{-}\right)$.

On the other hand, for the saddle-point solutions corresponding to (5.21), we have

$$
\operatorname{Re}\left[\frac{2 \pi^{2}}{\hbar} i S_{0}\right]=-(2 n+1) P\left(\tilde{q}_{+}, \tilde{q}_{-}\right) .
$$

From this result, we could say that the saddle contribution with $n \geq 1$ represents that from $n$ instantons. They are exponentially suppressed compared to the leading $n=0$ contribution. These saddle points for $n \geq 1$, however, do not contribute as saddles in the integral given above to evaluate the propagator due to the path that is chosen as the Lefschetz thimble of the $n=0$ saddle point.

\section{SUMMARY AND DISCUSSIONS}

In this paper, cosmology driven by SYM theory is studied in the FRW space-time. Here all the SYM fields are integrated out and the vacuum expectation value of their energy-momentum tensor $\left\langle T_{\mu \nu}^{\mathrm{SYM}}\right\rangle$ is given by holographic method. Based on the equations of motion with this $\left\langle T_{\mu \nu}^{\mathrm{SYM}}\right\rangle$, we introduced a new simple effective action that could reproduce the equations of motion of the theory.

The SYM theory provides two kinds of terms in the action, the loop correction term and a radiation term. The gravitational part includes the four-dimensional cosmological constant $\Lambda_{4}$. The value of $\Lambda_{4}$ is restricted to be positive in order to realize the inflationary universe at large scale factor $a_{0}$. In the present case, however, it is not completely free. When SYM theory is included, $\Lambda_{4}$ is bounded from above, so the expansion rate at large $a_{0}$ is modified by the loop correction of the SYM fields.

In the region of small $a_{0}$, the radiation plays an important role. Although the magnitude of the radiation is arbitrary, there appears a lower bound of $a_{0}$. Further, a small scale universe with the radiation could be born and appear at large $a_{0}$ after a quantum tunneling. Here, this phenomenon is studied through two quantum cosmological methods for minisuperspace of gravity.

One is to solve the WDW equation. Here the WDW equation is easily given by considering our effective action introduced as mentioned above. In this sense, our effective action is very useful to study the quantum mechanics of the theory. The tunneling probability is calculated by imposing an appropriate boundary condition at large $a_{0}$ for both cases of $C=0$ and $C>0$.

The other is to calculate the same quantity by the Lorentzian path integral according to the method proposed by [14]. This method is useful when the radiation is absent. The result coincides with the solution of the WDW equation.

On the other hand, it is difficult to proceed with the calculation in terms of the effective action used for the WDW equation when the radiation exists. So we consider a simplified model in which the potential in the WDW equation is approximated by the harmonic form. In this case, we could show a Lefschetz thimble as the unique path for the tunneling propagator. The calculation is performed according to the steepest descent method, and the result coincides with the solution of the WDW equation with the same harmonic potential. We should notice that, for the harmonic potential, we have many saddle points in the complex lapse $\mathcal{N}$ plane. However, the path, which contributes to the Lorentzian path integral, has only one saddle that corresponds to the tunneling.

Although, here, we concentrated on the tunneling amplitude, there are other kinds of propagators whose initial and final values of $a_{0}$ are different from those of the tunneling case. The situation also depends on the form of potential. It is characterized by the radiation and $\Lambda_{4}$. We will discuss in the future such various kinds of propagators. 


\section{ACKNOWLEDGMENTS}

One of the authors (M. T.) is supported in part by the JSPS Grant-in-Aid for Scientific Research, Grant No. 16K05357, and he is grateful for helpful discussions with Yuya Tanizaki.

\section{APPENDIX A: THE WHEELER-DEWITT EQUATION}

At first, we give the Wheeler-DeWitt equation used to obtain the wave function of the Universe. (3.4) is written as

$$
\begin{gathered}
S=\int d t L, \\
L=\mathcal{N} a_{0}^{3}\left(\frac{3}{a_{0}^{2}}\left(-\frac{\dot{a}_{0}^{2}}{\mathcal{N}^{2}}+k\right)-\Lambda_{\text {eff }}\right) v_{3}, \quad v_{3}=\frac{V_{3}}{\kappa_{4}^{2}},
\end{gathered}
$$

where

$$
\begin{aligned}
\Lambda_{\text {eff }}=3 \lambda_{-} & =3 \frac{1-\sqrt{1-4 \tilde{\alpha}^{2}\left(\frac{\Lambda_{4}}{3}+\tilde{\alpha}^{2} \frac{4 C}{a_{0}^{4}}\right)}}{2 \tilde{\alpha}^{2}}, \\
p_{a} & =\frac{\partial L}{\partial \dot{a}_{0}}=-\frac{6}{\mathcal{N}} a_{0} \dot{a}_{0} v_{3} .
\end{aligned}
$$

Then

$$
\begin{aligned}
H & =p_{a} \dot{a}_{0}-L \\
& =-\frac{\mathcal{N}}{12 a_{0} v_{3}}\left(p_{a}^{2}+12 a_{0}^{4} v_{3}^{2}\left(\frac{3}{a_{0}^{2}} k-\Lambda_{\text {eff }}\right)\right) \\
& =\mathcal{N} \hat{H},
\end{aligned}
$$

where

$$
\hat{H}=-\frac{1}{12 a_{0} v_{3}}\left(p_{a}^{2}+12 a_{0}^{4} v_{3}^{2}\left(\frac{3}{a_{0}^{2}} k-\Lambda_{\text {eff }}\right)\right) .
$$

Then we have

$$
S=\int d t\left(p_{a} \dot{a}_{0}-\mathcal{N} \hat{H}\right)
$$

This indicates that the lapse function $\mathcal{N}$ is a Lagrange multiplier providing the constraint

$$
\hat{H}=0 .
$$

This is written as a quantized form by using

$$
p_{a} \rightarrow-i \frac{\partial}{\partial a_{0}}
$$

as

$$
\left(-\frac{\partial^{2}}{\partial a_{0}^{2}}+12 a_{0}^{2} v_{3}^{2}\left(3 k-a_{0}^{2} \Lambda_{\text {eff }}\right)\right) \Psi\left(a_{0}\right)=0 .
$$

This equation could be applied to the region where a classical solution for $a_{0}(t)$ is forbidden. Below, by changing the variable in this equation, we show some numerical results for the tunneling process under appropriate boundary conditions.

\section{APPENDIX B: DERIVATION OF EQ. (3.3)}

The result (3.3) is obtained as follows by assuming the form of $L_{\text {SYM }}^{\text {eff }}$ as

$$
L_{\mathrm{SYM}}^{\mathrm{eff}}=h_{0}\left(a_{0}\right)+\frac{\dot{a}_{0}^{2}}{\mathcal{N}^{2}} h_{2}\left(a_{0}\right)+\frac{\dot{a}_{0}^{4}}{\mathcal{N}^{4}} h_{4}\left(a_{0}\right) .
$$

This form would be supported by the analyticity and $g_{0 i}=0$ gauge (3.1). By substituting this into (3.2), we find from $\delta S / \delta \mathcal{N}=0$,

$$
\begin{aligned}
& \left\{\frac{1}{\kappa_{4}^{2}}\left(3 a_{0}\left(\frac{\dot{a}_{0}^{2}}{\mathcal{N}^{2}}+k\right)-a_{0}^{3} \Lambda_{4}\right)\right. \\
& \left.\quad+a_{0}^{3}\left(h_{0}-\frac{\dot{a}_{0}^{2}}{\mathcal{N}^{2}} h_{2}-3 \frac{\dot{a}_{0}^{4}}{\mathcal{N}^{4}} h_{4}\right)\right\} \delta \mathcal{N}=0 .
\end{aligned}
$$

This is compared with (2.5) for $\mathcal{N}=1$ gauge,

$$
\lambda \equiv\left(\frac{\dot{a}_{0}}{a_{0}}\right)^{2}+\frac{k}{a_{0}^{2}}=\frac{\Lambda_{4}}{3}+\frac{\kappa_{4}^{2}}{3}\left\langle T_{00}^{\mathrm{SYM}}\right\rangle,
$$

where

$$
T_{00}^{\mathrm{SYM}}=\rho=\frac{3 \alpha}{16}\left(\frac{4 C}{a_{0}^{4}}+\lambda^{2}\right), \quad \lambda=\frac{\dot{a}_{0}^{2}}{a_{0}^{2}}+\frac{k}{a_{0}^{2}} .
$$

Then we have

$$
\begin{gathered}
h_{0}=-\frac{3 \alpha}{16}\left(\frac{4 C}{a_{0}^{4}}+\frac{k^{2}}{a_{0}^{4}}\right), \\
h_{2}=\frac{3 \alpha}{8} \frac{k}{a_{0}^{4}}, \\
h_{4}=\frac{\alpha}{16} \frac{1}{a_{0}^{4}} .
\end{gathered}
$$

Plugging this into (B1) we find (3.3), which is expressed for general $\mathcal{N}$. 


\section{APPENDIX C: PICARD-LEFSCHETZ METHOD}

In this appendix, let us introduce the Picard-Lefschetz method. $^{5}$ In the path-integral formalism, the partition function is defined by

$$
Z=\int_{\mathcal{M}} d \Phi \exp (\mathcal{F}(\Phi) / \hbar)
$$

where $\mathcal{M}$ is the target space of parameters $\Phi$. Since this integral is, in general, a multidimensional oscillatory integral, we need a technique to consider about it. Here we use the Lefschetz-thimble method [23,24].

The basic idea is to deform the integration contour $\mathcal{M}$ into steepest descent cycles inside its complexified space $\mathcal{M}_{\mathbb{C}}$ by using the Cauchy theorem when $\mathcal{F}$ is holomorphic. We denote the holomorphic coordinate of $\mathcal{M}_{\mathbb{C}}$ as $\Phi=\left(z^{1}, \ldots, z^{n}\right)$, and the set of saddle points as

$$
\Sigma=\left\{z_{\sigma}\right\}:=\left\{\frac{\partial \mathcal{F}}{\partial z^{i}}=0\right\} .
$$

Using the Kähler metric on $\mathcal{M}_{\mathbb{C}}, d s^{2}=g_{i \bar{j}} d z^{i} \otimes d \overline{z^{j}}$, we define the gradient flow by

$$
\frac{d z^{i}}{d t}=g^{i \bar{j}}\left(\frac{\partial \overline{\mathcal{F}}}{\partial \overline{z^{j}}}\right)
$$

As an important property of this differential equation, we have

$$
\frac{d \mathcal{F}}{d t}=|\partial \mathcal{F}|^{2} \geq 0
$$

\footnotetext{
${ }^{5}$ The notation of this appendix owes to [22].
}

Therefore, along the flow line, the real part of the free energy increases while its imaginary part stays constant. This means that we can define the steepest descent and ascent cycles associated with each saddle point $z_{\sigma}$ by this gradient flow. Using solutions of the gradient flow $z(t)$, they are defined as

$$
\begin{aligned}
& \mathcal{J}_{\sigma}=\left\{z(0) \mid z(t) \rightarrow z_{\sigma}, t \rightarrow-\infty\right\}, \\
& \mathcal{K}_{\sigma}=\left\{z(0) \mid z(t) \rightarrow z_{\sigma}, t \rightarrow+\infty\right\} .
\end{aligned}
$$

These are called Lefschetz thimbles and dual thimbles. They are dual quantities in terms of the intersection pairing $\langle\cdot, \cdot\rangle$, i.e., $\left\langle\mathcal{J}_{\sigma}, \mathcal{K}_{\tau}\right\rangle=\delta_{\sigma \tau}$, which means that one can decompose $\mathcal{M}$ in terms of $\mathcal{J}_{\sigma}$ as

$\int_{\mathcal{M}} d \Phi \exp (\mathcal{F}(\Phi) / \hbar)=\sum_{\sigma \in \Sigma}\left\langle\mathcal{M}, \mathcal{K}_{\sigma}\right\rangle \int_{\mathcal{J}_{\sigma}} d^{n} z \exp (\mathcal{F}(\Phi) / \hbar)$.

If all $\operatorname{Re}\left(\mathcal{F}\left(\Phi_{\sigma}\right)\right)$ are different from each other in the limit $\hbar \rightarrow 0$, we replace the integral by the saddle-point approximation. Then we obtain at the leading order that

$$
Z=\sum_{\sigma}\left\langle\mathcal{M}, \mathcal{K}_{\sigma}\right\rangle \exp \left(\mathcal{F}\left(z_{\sigma}\right) / \hbar\right)
$$

We can summarize the necessary steps of the mean-field approximation with the sign problem as follows:

(1) Complexify the target space $\mathcal{M}$ to $\mathcal{M}_{\mathbb{C}}$, and find the saddle points $z_{\sigma}$ by solving the equation $\partial \mathcal{F}=0$ in $\mathcal{M}_{\mathbb{C}}$.

(2) Solve the gradient flow (C3), and construct Lefschetz thimbles $\mathcal{J}_{\sigma}$ and dual thimbles $\mathcal{K}_{\sigma}$.

(3) Pick up the saddle point $z_{\sigma}$ that has the minimal free energy $\operatorname{Re}\left(\mathcal{F}\left(z_{\sigma}\right)\right)$ with nonzero intersection number $\left\langle\mathcal{M}, \mathcal{K}_{\sigma}\right\rangle$.
[1] K. Ghoroku, M. Ishihara, and A. Nakamura, Phys. Rev. D 74, 124020 (2006); 75, 046005 (2007).

[2] J. Erdmenger, K. Ghoroku, and R. Meyer, Phys. Rev. D 84, 026004 (2011).

[3] J. Erdmenger, K. Ghoroku, R. Meyer, and I. Papadimitriou, Fortsch. Phys. 60, 991 (2012).

[4] M. V. Fischetti, J. B. Hartle, and B. L. Hu, Phys. Rev. D 20, 1757 (1979).

[5] K. Ghoroku, R. Meyer, and F. Toyoda, Phys. Rev. D 96, 086011 (2017).

[6] A. Awad, Phys. Rev. D 93, 084006 (2016).

[7] A. O. Barvinsky, A. Yu. Kamenshchik, and D. V. Nesterev, Eur. Phys. J. C 75, 584 (2015).
[8] A. O. Barvinsky and A. Yu. Kamenshchik, J. Cosmol. Astropart. Phys. 09 (2006) 014; Phys. Rev. D 74, 121502 (2006).

[9] N. Bilic, Phys. Rev. D 93, 066010 (2016).

[10] P. Binetruy, C. Deffayet, U. Ellwanger, and D. Langlois, Phys. Lett. B 477, 285 (2000).

[11] D. Langlois, Phys. Rev. D 62, 126012 (2000); D. Langlois and L. Sorbo, Phys. Rev. D 68, 084006 (2003).

[12] T. Shiromizu, K. Maeda, and M. Sasaki, Phys. Rev. D 62, 024012 (2000).

[13] M. Sasaki, T. Shiromizu, and K. Maeda, Phys. Rev. D 62, 024008 (2000); K. Maeda, S. Mizuno, and T. Torii, Phys. Rev. D 68, 024033 (2003). 
[14] J. Feldbrugge, J.-L. Lehners, and N. Turok, Phys. Rev. D 95, 103508 (2017).

[15] J. J. Halliwell and J. Louko, Phys. Rev. D 39, 2206 (1989).

[16] J. Feldbrugge, J.-L. Lehners, and N. Turok, Phys. Rev. D 97, 023509 (2018).

[17] J. D. Dorronsoro, J. J. Halliwell, J. B. Hartle, T. Hertog, and O. Janssen, Phys. Rev. D 96, 043505 (2017).

[18] K. Ghoroku and A. Nakamura, Phys. Rev. D 87, 063507 (2013).

[19] S. de Haro, S. N. Solodukhin, and K. Skenderis, Commun. Math. Phys. 217, 595 (2001).
[20] M. Bianchi, D. Z. Freedman, and K. Skenderis, Nucl. Phys. B631, 159 (2002).

[21] C. Fefferman and C. R. Graham, in Elie Cartan et les Mathématiques d'aujourd'hui (Societe Mathematique de France, Paris, 1985), p. 95.

[22] Y. Tanizaki and M. Tachibana, J. High Energy Phys. 02 (2017) 081.

[23] E. Witten, AMS/IP Stud. Adv. Math. 50, 347 (2011).

[24] M. Cristoforetti, F. Di Renzo, A. Mukherjee, and L. Scorzato, Proc. Sci. LATTICE2013 02 (2014) 197. 